\title{
Analysis of Spatial-temporal Dynamics and Influencing Factors of County Economic Disparity: A Case Study of Gansu Province
}

\author{
Yan Han, Yuan Zhang, Meiling Deng \\ Department of Economics and Management \\ Lanzhou Jiaotong University \\ Lanzhou China
}

\begin{abstract}
This paper uses the analytical framework of exploratory spatial-temporal data analysis (ESTDA) to analyze the spatial-temporal dynamics and influencing factors of countylevel economic disparities in Gansu Province from 1995-2015. The results are as follows: Through the analysis of spatial-temporal evolution, it is found that the regional economy of Gansu Province has a local spatial differentiation while the spatial agglomeration area is expanding. The phenomenon of club convergence has emerged in regional economic development, and the regional economy has obvious path dependence characteristics. The spatial regression model was used to analyze the factors affecting regional economic differences in Gansu Province. Among them, the degree of decentralization and marketization have greater impact on spatial differentiation.
\end{abstract}

Keywords-Exploratory spatial-temporal data analysis; Agglomeration; Spatial Markov chain; Influencing factors

\section{INTRODUCTION}

As a geographical phenomenon with unbalanced development, regional economic differences are not only an important topic for academic research, but also problems that cannot be ignored in the process of regional economic development. The research on regional economy can be traced back to the 1950s, and gradually formed a theory of neoclassical theory, inverted U-shaped development theory, growth pole theory, core edge theory, imbalance theory, etc. The subject perspective is based on the multidisciplinary comprehensive intersection of regional economics, econometrics, environmental science, and geography [1-6]; the research scale includes global, national, provincial (state), municipal, county, etc. The provincial scale is the main trend, and it is gradually becoming smaller. This is because the differences of small-scale spatial units are more sensitive to fluctuations in the economic environment; research methods range from single indicators to multiple indicators, from measurement models to comprehensive analysis, and from single scales to Multi-scale comprehensive transformation, spatial measurement methods have gradually become the mainstream research methods; the main factors affecting the differences are globalization, policy tilt, decentralization and urbanization [9], resource and environment, industry basis, location factors [7-10], spatial neighbors Effects, special factors of the provincial border area
[3].The above studies provide useful analytical approaches for understanding regional economic differences.

A large number of empirical studies have shown that spatial dependence and heterogeneity are ubiquitous and not an exception. Economic development differences themselves coexist with spatial patterns of temporal dynamics and spatial patterns of temporal behavior. On the other hand, more and more scholars have noticed that the temporal and spatial properties of data are equally important, but the existing research methods focus on only one of them, namely spatial type analysis (formal analysis) and time series analysis (process analysis) is often separated [11-12]. However, the fact that cannot be ignored is that the dynamic process of regional economic development in empirical research includes two dimensions of time and space. Based on the effective integration of EDA (integration of spatial elements) and ESDA (integration of time factors), Rey proposed the framework of exploratory spatial data analysis (ESTDA), and the coupling and visual display of spatial-temporal correlation through the introduction of graph theory is a more successful exploration.

Based on the ESTDA analysis framework, this paper takes Gansu Province as the research object and takes the county area as the study scale to analyze the spatial-temporal evolution and change process of regional economic differences, and adopts three models: linear regression model, spatial lag regression model and spatial error regression model, to analyze the factors and to provide useful help for the coordinated development of regional economy.

\section{OVERVIEW OF THE STUDY AREA}

Gansu Province is located in the northwest fortress of China and is an important channel. As of the end of 2015, Gansu Province has a land area of 453,700 square kilometers. It has jurisdiction over 14 prefecture-level cities and 58 counties with a total population of $25,995,500$ people and a urbanization rate of $43.19 \%$.This paper uses 87 counties (districts) of Gansu Province as the basic research unit for the analysis of regional economic differences and spatial pattern evolution, and divides the entire province into four regional units 1: Hexiregion, the central region of Gansu, Southeast Yunnan, and the plateau margin according to geographical conditions.

This paper is sponsored by Gansu Science and Technology Agency Soft Science Project. (Number: 1504ZKCA017-3); Lanzhou Science and Technology Plan Project (number: 2014-1-249) 


\section{RESEARCH METHODS}

\section{A. Differences and agglomeration indicators}

This paper uses the Global Theil and Moran's I indices to measure the relationship between regional economic differences and spatial dependence over time. The Global Theil coefficient, which measures global relative differences, is calculated as follows:

$$
\mathrm{T}=\sum_{i=1}^{n} S_{i, t}\left[\log S_{i, t}-\log (1 / n)\right]
$$

The degree of spatial agglomeration is measured using the global autocorrelation Global Moran' I. The formula is as follows:

$$
I=\frac{n}{s_{0}} \sum_{i} \sum_{j} \frac{w_{i, j}\left(x_{i, j}-\overline{x_{t}}\right)\left(x_{j, t}-\overline{x_{t}}\right)}{\left(x_{i, t}-\overline{x_{t}}\right)^{2}}
$$

where $S_{i, t}$ is the proportion of the province's GDP in the $\mathrm{t}$ year $\mathrm{i}$ region, $\mathrm{N}=87, X_{i, t}$ for the county $\mathrm{i}$ in the $\mathrm{t}$ year of GDP per capita and the ratio of the province's per capita GDP, $S_{\mathrm{o}}$ is the sum of all elements in the weighting matrix for the space, $\overline{x_{t}}$ is the mean of a random variable $x_{t}$ for $\mathrm{t}$ years, and $x_{i, t}, x_{j, t}$ the observed value of county iand jin the t year, $w_{i j}$ for spatial weight matrix elements; $I \in[-1,1]$, The closer to 1 indicates that the positive space agglomeration is stronger, and vice versa.

The local spatial autocorrelation coefficient measures the degree of dispersion of each spatial unit and identifies the spatial form of the agglomeration area to fully reflect the changing trend of regional economic spatial differences. Calculated as follows:

$$
I i=\frac{(n-1) z_{i} \sum_{j} w_{i, j} z_{j}}{\sum_{j} z_{j}^{2}}
$$

The values of $I_{i}$ and $Z_{i}$ can indicate the clustering type of the location $i$ and its neighbors, respectively, which are high clustering, low concentration, high and low concentration, and low concentration.

\section{B. Spatial Markov chain}

Markov chain is an effective method to analyze the convergence of regional economic growth clubs. It discretizes the data of continuous attributes of geographical phenomena at different times, converts them into $\mathrm{k}$ types through the classification of numerical values, and calculates the probabilities of each type. Distribution and changes, which approximate the evolution of things as the Markov process [3].

The spatial Markov chain is a method combined with the concept of "spatial lag" to make up for the ignorance of the traditional Markov chain method for ignoring the spatial interaction of regional units [3] .Spatial Markov transition probability matrix Decomposes a conventional $\mathrm{k} \times \mathrm{k}$ Markov matrix into $\mathrm{k} \mathrm{k} \times \mathrm{k}$ conditional transition probability matrices. For the k-th conditional matrix, the element $(\mathrm{k})$ represents the region at $\mathrm{t}$ The spatial delay type $\mathrm{k}$ of the year is a background condition, and the year belongs to the space transition probability that the type $\mathrm{i}$ is transferred to the type $\mathrm{j}$ in the next year.

$$
\begin{aligned}
& M_{1}=\left[\begin{array}{ccc}
m_{(1,1 \mid 1)} & \cdots & m_{(1, k \mid 1)} \\
\vdots & \ddots & \vdots \\
m_{(k, 1 \mid 1)} & \cdots & m_{(k, k \mid 1)}
\end{array}\right]_{k * k} \cdots M_{k}= \\
& {\left[\left.\begin{array}{ccc}
m_{(1,1 \mid k)} & \cdots & m_{(1, k \mid 1 k)} \\
\vdots & \ddots & \vdots \\
m_{(k, 1 \mid k)} & \cdots & m_{(k, k \mid k)}
\end{array}\right|_{k * k}\right.}
\end{aligned}
$$

C. Spatial Regression Analysis

In order to better reveal the dynamic mechanism of regional economic development and differences in Gansu Province, this paper adopts the traditional linear regression model and spatial regression model analysis to conduct quantitative research, and compares the significance of several regression models so as to better conduct the influencing factors. Quantitative analysis.

\section{SOURCES OF DATA}

The original data of this paper comes from the $<<$ Statistical Yearbook of Gansu Province $>$ (2002-2016), and the data in $<<$ China City Statistical Yearbook $>>$ in the corresponding years. The 15-year period from 2002-2016 was selected as the time series spectrum, 87 spatial units, and 1305 observations. The GDP per capita is used to represent the level of economic development in the county; taking into account the availability of data, five indicators are selected, namely, financial expenditure (FE), total retail sales of consumer goods (MAR), total fixed-asset investment (FIX), and proportion of industrial output(IND) and(SAVING)for urban and rural residents respectively represent government decentralization, degree of marketization, regional policy tilt, industrial development level, and residents' storage capacity.

\section{RESEARCH RESULTS}

\section{A. Evolution of County Economic Disparity and Agglomeration}

Since 2002, the economic development of Gansu Province has been rapidly increased, and the per capita GDP has increased from 4,768 yuan to 27,643 yuan from 2002 to 2016. The average annual increase has been large, but there has been an uneven regional economic development. As can be seen from the figure, the Global Theil value of county per capita GDP in Gansu Province has been declining since 2002, and has dropped from 0.133 to 0.022 , indicating that the relative economic disparity in Gansu counties has continuously decreased during this period. Using GeoDA to calculate Moran's I, the global autocorrelation coefficient of per capita GDP in counties of Gansu Province from 2002 to 2016, we further explored the spatial correlation of county disparities in Gansu Province from 2002 to 2016. As shown in the figure, the overall autocorrelation Moran's I index of Gansu Province is positive and fluctuates, indicating that there is a positive spatial autocorrelation in per 
capita GDP of Gansu Province. From 2002 to 2004, the index dropped from 0.427 to 0.306 . It rebounded slightly to 0.326 in 2005. In 2005-2008, it maintained a declining trend and fell to 0.219 in 2008. From 2008 to 2010 , it continued to rise to 0.497 , and it decreased to 2002. 0.462, continued to rise in 2002-2016, reaching 0.627 in 2006 . In the study period, the distribution areas of the research units with the same degree of economic development in Gansu Province showed a concentrated trend, and the correlation fluctuations were large. In 2016, the overall Moran index reached 0.627 , and the agglomeration trend was obvious.

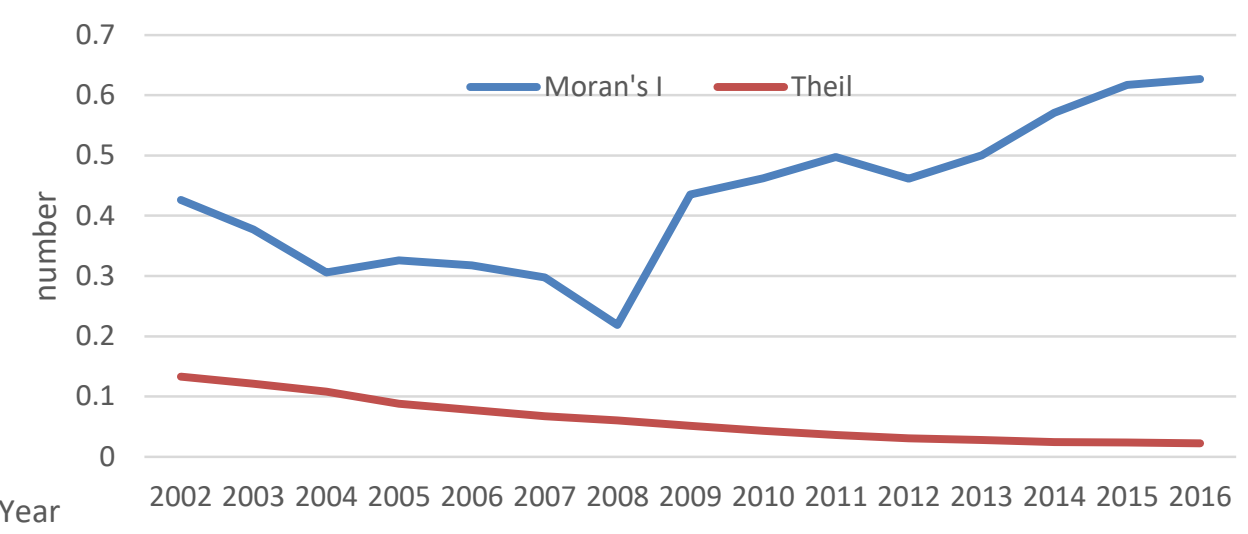

Fig. 1 The annual variation of inequality and agglomeration level

In order to further analyze the differences in the county economy in Gansu, we usesGeoDa to plot the LISA local spatial agglomeration maps for 2002, 2007, 2012, and 2016. As shown in the figure, the county economy in Gansu Province has a clear agglomeration trend. The higher level of economic development is concentrated in Subei Mongolian Autonomous County, Guazhou County, Dunhuang City, Axe Kazak Autonomous County, SunanYugu Autonomous County and other regions. There are obvious low-lying areas in the southeastern part of Gansu Province, which are economically backward areas and are mainly concentrated in Tongyu County, Wushan County,
Longxi County, Ganxian County, Gangu County, Ganxian County, Qinan County, Suichang County, and LintanCounty, Kangle County, Jingning County, Diebu County, Guanghe County, and Li County.

2002
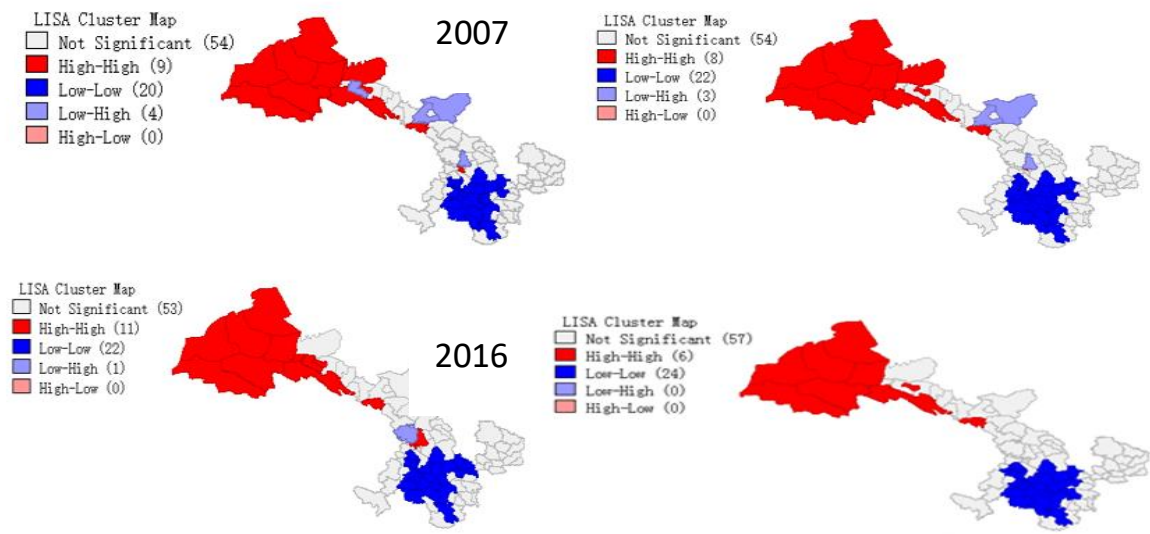

Fig. 2 Partial LISA gathering 


\section{B. Spatial Markov Chain Analysis}

The idea of space Markov chain is to consider the spatial neighborhood state of each area and analyze the spatial and temporal movement characteristics of regional units. This paper adopts the quartile method to divide the per capita GDP of the region into four types: low level, medium-low level, mediumhigh level, and high level. The division of spatial lag is the same as the division standard of per capita GDP. The spatial Markov chain transfer matrix is used to analyze the characteristics of long-term agglomeration or dispersion of regional economic levels in 87 different counties of 87 counties in Gansu Province, which provides a spatial solution for the influence of regional spatial relationships on regional economic growth convergence explanation. Table 1 shows the transitional probabilities of the economic development levels reflected by the GDP per capita in different regions of Gansu Province from 2002 to 2016. Ti and $\mathrm{Ti}+1$ indicate the economic level of the study area in year $\mathrm{i}$ and year $i+1$, respectively.

TABLE I TRANSITION PROBABILITY MATRIX OF SPATIAL MARKOV CHAINS

\begin{tabular}{llllll}
\hline spatial lag & ti/ti+1 & Low & Low-middle & High-middle & High \\
\hline \multirow{2}{*}{ Low } & Low & 0.86 & 0.14 & 0 & 0 \\
& Low-middle & 0.111 & 0.758 & 0.131 & 0 \\
& High-middle & 0 & 0.13 & 0.815 & 0.056 \\
& High & 0 & 0 & 0.273 & 0.727 \\
Low-middle & Low & 0.853 & 0.147 & 0 & 0 \\
& Low-middle & 0.084 & 0.822 & 0.084 & 0.009 \\
& High-middle & 0 & 0.146 & 0.805 & 0.049 \\
& High & 0 & 0.026 & 0.158 & 0.816 \\
High-middle & Low & 0.902 & 0.098 & 0 & 0 \\
& Low-middle & 0.083 & 0.75 & 0.167 & 0 \\
& High-middle & 0.01 & 0.08 & 0.84 & 0.07 \\
& High & 0 & 0 & 0.093 & 0.907 \\
Ligh & Low & 0.881 & 0.119 & 0 & 0 \\
& Low-middle & 0.174 & 0.739 & 0.087 & 0 \\
& High-middle & 0 & 0.017 & 0.833 & 0.15 \\
& High & 0 & 0 & 0.05 & 0.95 \\
\hline
\end{tabular}

From the space Markov chains we can see:

(1) The level of county economic development in Gansu Province is most likely to maintain its own state, with the value on the diagonal being much larger than the value on the nondiagonal line. The relatively large values at both ends of the diagonal indicate that the low and high levels are relatively more stable. Even in different neighborhood states, the probability of a county remaining in the initial state in the future is far greater than the probability of shifting to any other category. For example, the probability of a high-level region maintaining its own state in the low-, medium-, medium-, high-, and high-level neighborhood backgrounds is $73 \%, 81.6 \%, 90.7 \%$, and $95 \%$, respectively; the probability of a low-level region maintaining its own state is $86 \%$, respectively. $85.3 \%, 90.2 \%, 88.1 \%$. This shows that the polarization of the county economy in Gansu Province is relatively obvious, with the trend of spatial club convergence, and the regional internal economic difference structure has the characteristics of stability and long-term nature.

(2) The level of initial economic development in the region plays an important role in the future economic development.
There is a high probability of a shift from the medium level to the two ends, and the larger the horizontal gap, the greater the transition probability is. For example, in the adjacent type of transition, the low level is the neighborhood, and the highest possibility of high-to-medium level convergence is $27.2 \%$. The transition probability of all low-level to medium-high level and high-level is 0 .

(3) The regional background has a certain role in the dynamics and changes of regional economic convergence in Gansu Province. Under different neighborhood conditions, the transition probabilities of county-level GDP per capita are different. In general, neighboring high-level regions will increase the probability of upward shift; conversely, if they are neighbors of low-level areas, the probability of downward shift will increase. However, this rule is not obvious in the development of regional economy in Gansu Province. For example, in the context of high, medium, high, and lowneighborhood neighborhoods, the probability of shifting from high to medium levels to high levels is $15 \%, 7 \%$, and $0.9 \%$, respectively, but in the low-neighborhood context, this 
probability level is $5.6 \%$. It shows that the different regional backgrounds have different effects on the county economic development in Gansu Province, but they do not conform to the general laws.

\section{Analysis of Influencing Factors}

This article uses 2016 GDP per capita as a dependent variable, and takes fiscal expenditure, total retail sales of social consumer goods, total fixed asset investment, proportion of primary industry, and savings deposits of urban and rural residents as dependent variables, taking logarithm of variables, and applying classical regression and spatial Lag, spatial error model analyzes the degree of marketization, regional policy tilt, industrial development level, decentralization, and household savings have an impact on county economic differences.

As shown in Tables 2 and 3, comparing the fitting degree $\mathrm{R} 2$, the classical regression model is $71 \%$, the spatial regression model is more than $80 \%$, and the fitting effect is better than the linear regression model, and both the AI value and the $\mathrm{SC}$ value are reduced. Among them, the spatial lag model is most consistent with the objective reality, and the fitting degree of the linear regression model is improved by $11.6 \%$. Compared with the linear regression model, in the two spatial regression models, the marginal effect of variables has basically declined. This phenomenon is mainly due to the fact that the classical regression model does not consider spatial factors and ignores the spatial spillover effects of variables. After incorporating the spatial weight matrix into the spatial regression model, the marginal effect response of each variable is more realistic.

As shown in Tables 1 and 2, all variables passed the test with a $1 \%$ level of significance, indicating that the degree of decentralization, the degree of marketization, the tilt of regional policies, the level of industrial development, and the level of resident deposits are the key causes of economic disparities in counties.

Compared with the classical regression model, the fixedasset investment in the spatial regression model is significantly lower, indicating that after the spatial factors are added, the impact of the regional policy tilt on the county-level economic differences increases. The significant increase in the two indicators of household savings deposits and the proportion of industrial industries after the addition of spatial factors indicates that the classical regression model exaggerates the extent to which the degree of household savings deposits and the level of industrial development affect economic disparities. In the spatial regression model, the two indicators of fiscal expenditure and total retail sales of consumer goods are the most significant, indicating that the degree of decentralization and marketization have a greater impact on spatial differentiation. The marginal effect of fiscal expenditure on GDP per capita in the spatial lag model is -0.628 , which indicates that the degree of decentralization is negatively correlated with GDP per capita. Every additional unit of fiscal expenditure increases the per capita GDP by 0.628 . The marginal effect of total retail sales of consumer goods on GDP per capita is 0.426 , and the degree of marketization is positively correlated with GDP per capita.

TABLE II OLS RESULTS FOR CLASSIC LINEAR REGRESSION

\begin{tabular}{llcclll}
\hline Variable & Coefficient & $\begin{array}{c}\text { Std. } \\
\text { Error }\end{array}$ & $\begin{array}{c}\text { t- } \\
\text { Statistic }\end{array}$ & Probability & Test \\
\hline CONSTANT & 16.598 & 1.568 & 10.585 & 0.000 & R-squared & 0.726590 \\
$\ln ($ FE) & -0.855 & 0.171 & -5.008 & 0.000 & Adjusted R-squared & 0.710 \\
$\ln ($ MAR $)$ & 0.571 & 1.106 & 5.378 & 0.000 & F-statistic & 43.052 \\
$\ln ($ FAI $)$ & 0.205 & 0.063 & 3.242 & 0.002 & P & 0.000 \\
$\ln ($ IND) & 0.312 & 0.067 & 4.659 & 0.000 & AIC & 93.562 \\
$\ln ($ Saving) & -0.399 & 0.142 & -2.819 & 0.006 & SC & 108.357 \\
\hline
\end{tabular}


TABLE III RESULTS FOR THE TWO SPATIAL AUTOREGRESSIVE MODELS

\begin{tabular}{|c|c|c|c|c|c|c|}
\hline \multicolumn{4}{|c|}{ spatial lag regression model } & \multicolumn{3}{|c|}{ spatial error regression model } \\
\hline Variable & Coefficient & Std.Error & $\begin{array}{c}\text { Z- } \\
\text { value }\end{array}$ & Coefficient & Std.Error & Z-value \\
\hline W_Pcgdp & 0.473 & 0.061 & 7.793 & & & \\
\hline CONSTANT & 9.821 & 1.379 & 7.124 & 12.966 & 1.262 & 10.273 \\
\hline $\ln (\mathrm{FE})$ & -0.628 & 0.127 & -4.959 & -0.515 & 0.144 & -3.579 \\
\hline $\ln (\mathrm{MAR})$ & 0.426 & 0.080 & 5.323 & 0.422 & 0.091 & 4.623 \\
\hline $\ln (\mathrm{FAI})$ & 0.189 & 0.047 & 4.060 & 0.195 & 0.045 & 4.373 \\
\hline $\ln (\mathrm{IND})$ & 0.190 & 0.051 & 3.750 & 0.182 & 0.058 & 3.157 \\
\hline $\ln ($ Saving $)$ & -0.323 & 0.104 & -3.093 & -0.313 & 0.121 & -2.574 \\
\hline LAMBDA & & & & 0.716 & 0.078 & 9.167 \\
\hline R-squared & 0.843 & & & 0.834 & & \\
\hline Breusch-Pagan test & 2.230 & & & 4.655 & & \\
\hline Likelihood Ratio Test & 43.510 & & & 30.039 & & \\
\hline
\end{tabular}

\section{CONCLUSIONS AND POLICY RECOMMENDATIONS}

By introducing the spatial weight matrix, the spatialtemporal dynamics of the county-level economic differences in Gansu Province are analyzed. The indicators show that the county-level economic development level in Gansu Province has obvious spatial and positive spatial correlations, and there is a phenomenon of high agglomeration and low-low agglomeration. Through the analysis of the spatial Markov chain probability transfer matrix, it can be found that there are club convergence phenomenon in the development of county economy, with the trend of spatial club convergence, and the regional internal economic difference structure has the characteristics of stability and long-term, and the county economy has obvious path dependence feature. The spatial regression analysis shows that compared with the traditional OLS model, the space spillover effect of variables is eliminated after taking into account the spatial regression analysis of the spatial factors, making the marginal effect of each variable more real and effective. The regression results show that the degree of decentralization, degree of marketization, tilted regional policies, industrial development level, and residents' deposit level are important factors that cause differences in county economies.

\section{REFERENCES}

[1] Chen Peiyang and Zhu Xigang, Regional economic differences in China based on differentscales. Journal of Geographical Science, 2012 (08): 1085-1097.

[2] [Li Zaijun et al. Spatial Pattern of China's Economic Growth in the New Era. GeographyScience, 2016(08): 1134-1140.

[3] [Chen Peiyang and Zhu Xigang, Regional Economic Convergence in China: Spatial MarkovChain Analysis Based on County Scale. Geography Science, 2013(11): 1302-1308.

[4] Tang Xiumei, et al. Spatial Variation of the Per Capita GDP of the JingJin-Ji Region and ItsInfluencing Factors. Journal of Peking University (Natural Science), 2017(06):1089-1098

[5] Yang Dongyang, et al., Study on the Temporal and Spatial Evolution of County EconomicDisparity in the Central Plains Economic Zone. Regional Research and Development, 2015(05):29-33.

[6] Ye Xinyue, Li Jingjing and Cheng Yeqing, Multi-scale and multimechanism analysis of spatial-temporal dynamics of economic disparities in Zhejiang Province. Progress in Geography. 2014(09): 1177 1186.

[7] Zhang Xuebo et al. Evolution of spatial pattern of economic differences in the border areas ofChina's provinces. Economic Geography, 2015(07): 30-38.

[8] Chen Peiyang and Zhu Xigang, Spatial analysis of regional economic disparities and their dynamic mechanisms in Fujian Province. Economic Geography, 2011(08): 1252-1257.

[9] Du Xia, Qian Hongsheng and Wu Dianting, The spatial differentiation of the county economyin Shandong Province and its causes. Urban Issues, 2015(08): 97-103.

[10] Ji Xiaomei et al., Mechanism of spatial-temporal dynamics and change of county-level economic disparities in Fujian Province. Economic Geography, 2016(02): 36-44.

[11] YE X, R.S., A framework for exploratory space-time analysis of economic data. The Annalsof Regional Science, 2013. 1(50):315-339.

[12] Dong Yaning, Regional Economic Development Differences in Gansu Province Based on New Economic Geography Theory. Regional Research and Development, 2016(01): 87-90. 